\title{
MEDICINA DE ESTILO DE VIDA: EL ORIGEN DE UNA NUEVA ESPECIALIDAD MÉDICA
}

\author{
LIFESTYLE MEDICINE: THE ORIGIN OF A NEW MEDICAL ESPECIALITY \\ Jhony A. De La Cruz -Vargas\$,2,a,b,c, Nahúm García-García² , Luiz Sella²
}

La medicina evoluciona a pasos agigantados y también lo hacen las especialidades mas necesarias. Un articulo recientemente publicado hace referencia a las 5 nuevas especialidades en USA: Inmunooncologia, Medicina de Estilo de Vida, Nocturnistas, Informática Clínica y Medico Virtualista. La demanda actual y futura de estas nuevas especialidades va en aumento.

\section{¿Por que Medicina de Estilo de Vida ha llegado para quedarse como una atractiva y nueva especialidad del presente y del futuro?}

Alrededor del $80 \%$ de los costos de atención medica están relacionados con enfermedades crónicas, y el $80 \%$ de las enfermedades crónicas están relacionadas con las opciones del estilo de vida. Esto constituye un poderoso argumento para los especialistas en medicina de estilo de vida.

La elección de los alimentos de un paciente y su dieta, el ejercicio, el sueño, los niveles de estrés y la capacidad para relacionarse con los demás están bajo el alcance de un medico especialista en medicina de estilo de vida. Medicina de Estilo de Vida se ocupa de todos los aspectos de la vida de una persona.

La atención primaria y la medicina familiar son lugares naturales para los expertos en medicina de estilo de vida, pero también juega un rol fundamental en las clínicas de medicina de estilo de vida donde los pacientes requieren enfoques intensivos, cuando la necesidad es urgente.

El American Board of Lifestyle Medicine comenzó a ofrecer la certificacion en medicina de estilo de vida en octubre de 2017, en Tucson, Arizona. El International Board of Lifestyle Medicine junto a las organizaciones regionales son las encargadas de la certificacion en los 5 continentes.

En Perú y en Latinoamerica, Latin American Lifestyle Medicine Association, viene trabajando para impulsar y desarrollar medicina de estilo de vida como pilar fundamental en la practica medica cotidiana. En este entorno nace lifestyle medicine, un nuevo concepto de tratar a la persona de de manera integral, no solo la patologia por la que consulta.

Algunas de la razones por las que Medicina de Estilo de Vida viene creciendo exponencialmente en los ultimos 15 años como una nueva disciplina medica y se ha convertido en una atractiva propuesta como especialidad, son las siguientes:

1. La Epidemia de las "Enfermedades Cronicas" como principal causa de morbimortalidad. 40 millones de muertes por año a nivel mundial.

2. Las "Enfermedades Cronicas" surgieron no como consecuencia de una nueva mutacion genetica sino en relacion directa con los habitos y estilo de vida de las personas.

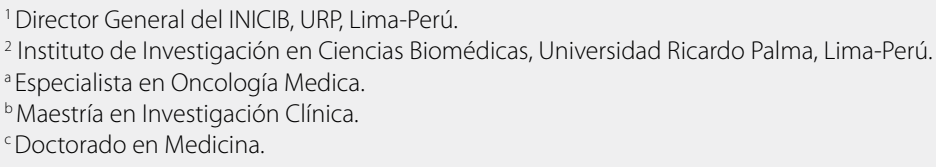

Citar como: Jhony A. De La Cruz -Vargas, Luiz Sella Nahúm García-García. Medicina de estilo de vida: El origen de una nueva especialidad médica. [Editorial]. Rev. Fac. Med. Hum. 2018;18(4):7-9. (Octubre 2018). DOI 10.25176/RFMH.v18.n4.1724 
3. Las "Enfermedades Cronicas" explican la gran mayoria de muertes prematurasy sus consecuencias en años de vida saludable perdidos en la poblacion, y esto impacta directamente en el desarrollo social y economico de los paises y sociedades.

4. Los costos en salud con el modelo actual resulta insostenible para los presupuestos de los paises, donde solo con obesidad, diabetes, cancer, ECV neurodegenerativas y otras enfermdades los gastos se tornan inviables.

5. Las enfermedades cronicas no solo producen enfermedad, muerte, años de vida saludable perdidos, perdida de capacidad productiva, sino que ademas afecta directamente la calidad de vida de las personas.

6. Medicina de estilo de Vida es el nuevo paradigma que propone atacar directamente las verdaderas causas de las enfermedades ( habitos del estilo de vida) y no solo el manejo de signos y sintomas de las enfermedades del modelo convencional.

7. La propuesta de fondo es cambiar de una medicina diagnóstica-terapeutica en su gran mayoria acostumbrada a manejo de enfermedad avanzada a Medicina de Estilo de Vida: Medicina preventiva y diagnostico temprano de las enfermdades.

8. Medicina de Estilo de Vida toma lo mejor de Medicina Preventiva y de Salud Publica, para bajarlo y llevarlo a las personas interesadas en cuidar y disfrutar su salud y no en manejar enfermedades (concepto clasico de los hospitales).

9. Medicina de Estilo de Vida es Medicina Basada en Evidencia, el cuerpo de evidecia cientifica disponible va en aumento, y posee un excelente perfil de seguridad (minimos o nulos efectos secundarios) y costo efectiva.

10. El concepto mas poderoso de alta repercusion en salud publica es que Medicina de Estilo de Vida ha demostrado "Alto poder terapeutico" y puede "Revertir las enfermedades cronicas", rompiendo paradignas de la ciencia medica moderna.

11. Medicina de Estilo de Vida posee estructura organica en los 5 contienentes, conviertiendose en la nueva disiciplina medica de carácter global.( https://lifestylemedicineglobal.org/)

12. Medicina de Estilo de Vida cuenta con la aprobacion de la American Medical Association (AMA), creandose en el 2004 el American College of Lifestyle Medicine. (https://www.lifestylemedicine.org/)
13. Actualmente existe presencia en mas de 30 paises a nivel mundial de la Alianza Global de Medicina de Estilo de Vida, en Latinoamerica esta representada por la Asociacion Latinomaericana de Medicina de Estilo de Vida. (www.lalma.co)

14. Se han dearrollado y publicado el core de 15 competencias para ejercer la Medicina de Estilo de Vida a nivel internacional.

15. Se genero el American Board of Lifestile Medicine(https://ablm.co/) para dirigir la Certificacion en Medicina de Estilo de Vida en USA y el International Board of Lifestyle Medicine( https:// iblm.co/) para el resto del mundo.

16. En Estados Unidos se han desarrollado consorcios universitarios especificos de Medicina de Estilo de Vida (LMED: http://lifestylemedicineeducation. org/)

17. Cada vez mas universidades en el mundo estan incorporando Medicina de Estilo de Vida tanto en el pregrado como en el posgrado.(Loma Linda University, Harvard, University of South Carolina, Universidad de Montemorelos, etc).

18. Existen disponibles a nivel internacional Maestrias en Medicina de Estilo de Vida. Los principales Centros de referencia en el mundo estan trabajando con Medicina de Estilo de Vida (John Hopkins, Mayo Clinic, Harvard, Loma Linda University, Teknon, etc.)

19. El American College of Lifestyle Medicine ha desarrollado fellowship en Medicina de Estilo de Vida.

20. En Peru, la Universidad Ricardo Palma es Pionera en promover y respaldar Medicina de Estilo de Vida en Peru. Recientemente se desarrolló con éxito el Il Congreso Latinoamericano de Medicina de Estilo de Vida ( www.lalmalifestylemedicinecongress.org) y la primera certificacion internacional en medicina de estilo de vida.

En Medicina de Estilo de Vida las personas son el "Centro de la atencion medica", el objetivo es empoderar a la poblacion en el cuidado de su salud, dejando de ser receptores pasivos de prescripciones medicas sino transformandolos en socios activos en la toma de decisones, fijando objetivos realisticos y modificando sus habitos para tener estilos de vida saludables y sostenibles.

El horizonte de lifestyle medicine como el origen de una nueva especialidad medica esta creciendo como un tsunami a nivel internacional. Es fundamental desarrollar estrategias para su incorporacion en los planes curriculares de pre y posgrado en las escuelas y 
facultades de medicina. Tenemos la responsabilidad y la oportunidad de ser pioneros en medicina de estilo de vida en Perú y Latinoamerica y no solo seguidores cuando todos ya lo hallan hecho.

Correspondencia: Jhony A. De La Cruz Vargas

Dirección: INICIB, Facultad de Medicina Humana, Edificio 1-208. 2do piso. Avenida Benavides 5440, Surco, Lima-Perú.

Teléfono: 708-0000/Anexo: 6016

Correo:jhony.delacruz@urp.edu.pe

\section{REFERENCIAS BIBLIOGRÁFICAS}

1. Sayburn Anna. Lifestyle medicine: a new medical specialty? BMJ 2018;363:k4442.

doi: https://doi.org/10.1136/bmj.k4442 (Published 25 October 2018)

2. Bodai BI, Nakata TE, Wong WT, et al. Lifestyle medicine: A brief review of its dramatic impact on health and survival. Perm J 2018;22:17-025.

DOI: https://doi.org/10.7812/TPP/17-025

3. Wayne S. Dysinger. MEDICAL EDUCATION Lifestyle Medicine Competencies for Primary Care Physicians. American Medical Association Journal of Ethics April 2013, Volume 15, Number 4: 306-310.

https://journalofethics.ama-assn.org/sites/journalofethics.ama-assn.org/ files/2018-05/medu1-1304.pdf

4. Jhony A. De La Cruz-Vargas, Wayne Dysinger, Stephan Herzog, Fabio dos Santos, Henry Villegas, Margarete Ezinwa. Lifestyle Medicine: working together to reverse the chronic disease epidemic in Latin America. [Editorial]. Rev. Fac. Med. Hum. 2017;17(1):10-12.

DOI: https://doi.org/10.25176/RFMH.v17.n1.742

5. Robert F Kushner, Jeffrey I Mechanick. Lifestyle Medicine an emerging new discipline. US Endocrinology, 2015;11(1):36-40

http://doi.org/10.17925/USE.2015.11.1.36

6. Sagner M1, Katz D, Egger G, Lianov L, Schulz KH, Braman M, Behbod B, Phillips E, Dysinger W, Ornish D. Lifestyle medicine potential for reversing a world of chronic disease epidemics: from cell to community. Int J Clin Pract. 2014 Nov;68(11):1289-92.

https://doi.org/10.1111/ijcp.12509

\section{Links de Universidades y Maestrias en Medicina de Estilo de Vida:}

1. https://lifestylemedicine.hmscme.com/

2. https://publichealth.Ilu.edu/academics/mph/mph-lifestyle-management-online

3. https://www.instituteoflifestylemedicine.org/?page_id=8

4. https://gradschool.creighton.edu/program/lifestyle-medicine-graduate-certificate

5. http://www.jr-lifestylemedicine.com/en/master-of-lifestyle-medicine/

\section{Indizado en: latindex}

http://www.latindex.org/latindex/ficha?folio=14280

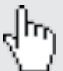

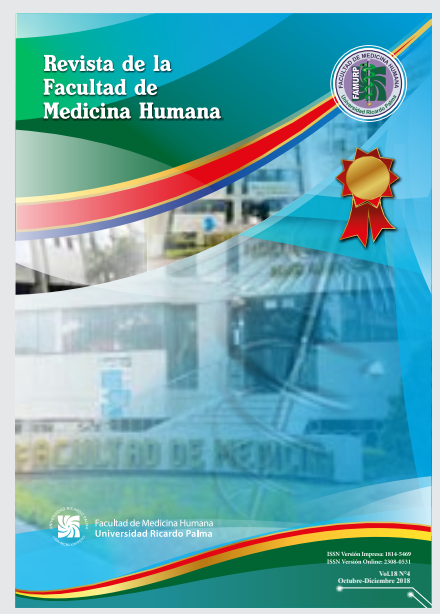

